\title{
1 Influence of an anionic polysaccharide on the 2 physical and oxidative stability of omega-3 \\ 3 nanoemulsions: Antioxidant effects of \\ 4 alginate
}

5

6

Journal: Food Hydrocolloids

Submitted: May 2015

*Department of Food Science, University of Massachusetts, Amherst, MA, 01003; Fax 413545 1262; Tel 413545 1019; email: mcclements@foodsci.umass.edu. 


\section{Abstract}

The aim of this study was to assess the impact of an anionic polysaccharide on the physical properties and chemical stability of omega-3 enriched nanoemulsions. Fish oil-inwater nanoemulsions stabilized with a non-ionic surfactant (Tween 80) were mixed with sodium alginate at several concentrations, and then their viscosity, creaming stability, particle size, microstructure, and oxidation were measured. The viscosity of the mixed systems $(2.5 \%$ w/w of oil) increased with polysaccharide addition, and was primarily governed by the alginate rather than the oil droplets. Droplet flocculation was observed at sodium alginate concentrations exceeding $0.05 \%(\mathrm{w} / \mathrm{w})$, which led to rapid creaming and an increase in droplet size due to coalescence. This effect was attributed to depletion flocculation arising from the exclusion of non-adsorbed polysaccharide molecules from the immediate vicinity of the droplet surfaces. Nevertheless, the addition of alginate $(0.1 \% \mathrm{w} / \mathrm{w})$ to the nanoemulsions decreased the rate and extent of lipid oxidation during storage. This effect was probably due to the ability of anionic groups on the alginate molecules to chelate pro-oxidant transition metals (such as iron) in the aqueous phase. This study highlights the potential of using alginate as a natural antioxidant in nanoemulsions; however, it also highlights the potential for this polysaccharide to promote physical instability. This information could be used to optimize the composition and structure of food matrices designed to improve the oxidative stability of polyunsaturated lipids.

Keywords: Nanoemulsions; omega-3 fatty acids; lemon oil; oxidation; sodium alginate; food matrix effects 


\section{Introduction}

Fish oils are naturally rich in omega-3 fatty acids, such as eicosapentaenoic acid (EPA) and docosahexaenoic acid (DHA), which are long chain polyunsaturated fatty acids (PUFAs). Omega-3 fatty acids play an important role in human nutrition, since their consumption has been associated with a reduced risk of cardiovascular diseases and cancer, improved brain development, and prevention of inflammatory related pathologies (Adkins \& Kelley, 2010; Lorente-Cebrian, et al., 2013; Shahidi \& Miraliakbari, 2004, 2005). Due to their reported health benefits, the food industry has an increasing interest in food fortification with fish oils (Ganesan, Brothersen, \& McMahon, 2014). However, there are challenges associated with their incorporation into food formulations due to their low water-solubility and poor chemical stability. Omega-3 fatty acids are highly susceptible to lipid oxidation, which can negatively impact the shelf life, nutritional value, and flavor of foods (Arab-Tehrany, et al., 2012; Sun, Wang, Chen, \& $\mathrm{Li}, 2011)$. Lipid oxidation is the result of a complex reaction between unsaturated fatty acids and oxygen-reactive species, which can be divided into initiation, propagation, and termination phases (Waraho, McClements, \& Decker, 2011).

In oil-in-water $(\mathrm{O} / \mathrm{W})$ emulsions, the oxidation of PUFAs is usually initiated by the interaction of lipid hydroperoxides located at the surface of the oil droplets with transition metals located in the surrounding aqueous phase (Berton-Carabin, Genot, Gaillard, Guibert, \& Ropers, 2013; Berton-Carabin, Ropers, \& Genot, 2014; McClements \& Decker, 2000). Transition metals are able to break down unsaturated lipids into alkyl radicals, but this reaction occurs extremely slowly and is therefore not considered to be the main cause of lipid oxidation (Akoh \& Min, 2008). The dominant reaction causing lipid oxidation is the decomposition of lipid hydroperoxides into peroxyl and alkoxyl radicals by transition metals, which are highly reactive species able to react with unsaturated lipids in the oil droplet surface. In turn, this reaction leads to the formation of lipid radicals that cause the propagation of the lipid oxidation reaction when they interact with other lipid molecules in their vicinity. The formation of lipid radicals eventually terminates when they react with other radicals due to the formation of non-radical compounds (McClements \& Decker, 2000). The rate and extent of lipid oxidation in emulsion-based food products is affected by several factors, including temperature, $\mathrm{pH}$, exposure to UV-light, and the ratio of antioxidant to pro-oxidant species present in the food matrix. Thus, there is a need to investigate the role of specific food 
constituents on lipid oxidation in food emulsions so as to develop more stable food products that are susceptible to lipid oxidation, such as those enriched with omega-3 fatty acids.

$\mathrm{O} / \mathrm{W}$ nanoemulsions are a type of emulsion-based delivery system that consists of small $(r<100 \mathrm{~nm})$ oil droplets dispersed within an aqueous continuous phase David Julian McClements and Rao (2011). Edible nanoemulsions are particularly suitable for the encapsulation, protection, and release of omega-3 fatty acids because of their good physical stability and high optical clarity (Walker, Decker, \& McClements, 2015). In addition, recent in vivo studies have shown that nanoemulsions lead to higher intestinal absorption of omega-3 fatty acids compared to conventional emulsions (Dey, Ghosh, Ghosh, Koley, \& Dhar, 2012). The incorporation of omega-3 fatty acids in nanoemulsions has been the focus of several research papers, confirming the feasibility to achieve highly stable delivery systems (Belhaj, Arab-Tehrany, \& Linder, 2010; Gulotta, Saberi, Nicoli, \& McClements, 2014). Nevertheless, there is currently a lack of information on the oxidative stability of omega- 3 enrich nanoemulsions once they are incorporated into food systems. Therefore, there is a need to study the potential interactions between omega-3 nanoemulsions and other ingredients present in food formulations that might alter their physicochemical stability.

Polysaccharides are commonly used as functional ingredients in the food industry to formulate emulsion-based foods, e.g., as stabilizing, thickening, or gelling agents (Phillips \& Williams, 2000). The addition of sufficiently high levels of polysaccharide to the aqueous phase of an emulsion inhibits creaming by thickening or gelling the aqueous phase. However, over a certain concentration range polysaccharides may actually promote physical instability in emulsions by promoting droplet flocculation through a bridging or depletion mechanism (Dickinson, 2003; McClements, 2015). Consequently, the amount of polysaccharide added to an emulsion as a functional ingredient must be carefully controlled to produce the desired textural properties and physical stability. Certain types of polysaccharides may also play other important roles in stabilizing emulsion-based foods. For example, studies have shown that certain types of anionic polysaccharide can inhibit the oxidation of emulsified lipids due to their ability to chelate metallic ions (Kishk \& Al-Sayed, 2007).

The specific aim of the present work was to study the influence of polysaccharide addition on the rheology, physical stability, and oxidative stability of omega-3 nanoemulsions. Nanoemulsions were prepared from an oil phase that consisted of a blend of fish oil and lemon oil. The fish oil was the source of omega-3 fatty acids, whereas the lemon 
111 oil was used to facilitate nanoemulsion formation by lowering the interfacial tension and 112 dispersed phase viscosity (McClements, et al., 2011). The impact of sodium alginate on the 113 rheology and physical stability of the emulsions was examined, whereas the impact of three

114 polysaccharides with different charge characteristics was used in the oxidation studies:

115 sodium alginate (anionic), chitosan (cationic) and methylcellulose (non ionic). Overall, the

116 objective of this research was to provide information about the potential beneficial or

117 detrimental effects of polysaccharide addition on the properties and stability of omega-3 fatty 118 acid enriched nanoemulsions.

\section{$119 \quad 2$ Material and methods}

\subsection{Materials}

Fish oil (Ropufa 30 n-3 food oil) was obtained from DSM Nutritional Products Ltd.

122 (Basel, Switzerland). The oil contained $101 \mathrm{mg}$ of EPA/g of oil, $148 \mathrm{mg}$ of DHA/g oil, and a 123 total of omega-3 PUFA of $312 \mathrm{mg} / \mathrm{g}$ of oil. Lemon oil (SC 020207, Lot: 2894308) used in this 124 work was kindly donated by International Flavors and Fragrances (Union Beach, N.J.). Iso125 octane, 2-propanol, methanol, buthanol and ethanol were obtained from Fischer Scientific 126 (MA, USA). $\mathrm{Fe}_{2} \mathrm{SO}_{4}$, ethylenediaminetetraacetic acid (EDTA), thiocyanate, trichloroacetic acid, thiobarbituric acid, butylated hydroxytoluene (BHT), ethanol, 1,1,3,3-

128 tetraethoxypropane (TEP), Tween 80, sodium acetate, acetic acid, sodium alginate, chitosan,

129 methylcellulose, and Nile Red dye were purchased at Sigma-Aldrich (St. Louis, MO). All

130 aqueous solutions were prepared using purified water from a Milli-Q filtration system.

\subsection{Methods}

\subsubsection{Nanoemulsion formation}

133 The lipid phase of nanoemulsions consisted of a mixture of fish oil and lemon oil (50:50 $134 \mathrm{w} / \mathrm{w}$ ). Both oils were mixed and stirred for $5 \mathrm{~min}$ at room temperature. The aqueous phase of 135 the nanoemulsions consisted of $100 \mathrm{mM}$ acetic-acetate buffer at $\mathrm{pH}$ 3.0. A coarse emulsion 136 was formed by mixing lipid phase $(10 \% \mathrm{w} / \mathrm{w})$, Tween $80(1 \% \mathrm{w} / \mathrm{w})$, and aqueous phase $(89 \%$ $137 \mathrm{w} / \mathrm{w}$ ) with a high-sheer blender for $2 \mathrm{~min}$ at 20,000 rpm. The resulting coarse emulsion was 138 then passed three times through a microfluidizer (model M110-P, Microfluidics, Newton, 139 MA) working at 15,000 psi to form a nanoemulsion. 


\subsubsection{Mixing experiments}

A sodium alginate stock solution was prepared by stirring the powdered polysaccharide ingredient $(2 \% \mathrm{w} / \mathrm{w})$ into acetic-acetate buffer $(100 \mathrm{mM}, \mathrm{pH} 3.0)$ until complete dissolution had occurred. Sodium alginate solution was used immediately without any further storage. A series of nanoemulsions containing different alginate concentrations $(0,0.01,0.02,0.05,0.1$, $0.25,0.5,0.75,1 \% \mathrm{w} / \mathrm{w})$ but the same oil $(2.5 \% \mathrm{w} / \mathrm{w})$ and surfactant $(0.25 \% \mathrm{w} / \mathrm{w})$ content were prepared by mixing sodium alginate stock solution $(2 \% \mathrm{w} / \mathrm{w})$, buffer solution $(100 \mathrm{mM}$ acetic-acetate, $\mathrm{pH}$ 3.0) and nanoemulsions together in different ratios. The systems were stirred for 5 minutes before physicochemical characterization.

\subsubsection{Rheological properties}

The rheological properties of the mixtures containing sodium alginate and nanoemulsions were measured using a dynamic shear rheometer (Kinexus, Malvern Instruments Ltd, Worcestershire, UK). In addition, sodium alginate solutions with the same polysaccharide concentration range as the mixed systems were also measured. A cup-and-bob measurement cell was used for all measurements: the bob had a diameter of $25 \mathrm{~mm}$ and the cup had a diameter of $27.5 \mathrm{~mm}$. The shear stress was measured as a function of shear rate from 0.1 to $100 \mathrm{~s}^{-1}$ at $25^{\circ} \mathrm{C}$. The apparent shear viscosity is also reported at $10 \mathrm{~s}^{-1}$, a rate that is similar to that experience by foods during mastication of semi-solid foods (Shama \& Sherman, 1973). The measurements were programmed and recorded using the instrument software (Kinexsus rSpace, Malvern Instruments Ltd., MA, USA).

\subsubsection{Creaming index}

Creaming of mixed systems $(10 \mathrm{~mL})$ was monitored at room temperature by visual observation and measurement with a ruler of the thickness $(\mathrm{mm})$ of the serum layer formed at the lower part of glass tubes $\left(\mathrm{H}_{\mathrm{S}}\right)$, expressed as a percentage of the total height of the emulsions in the tubes $\left(\mathrm{H}_{\mathrm{E}}\right)$ : Creaming Index $(\mathrm{CI})=100 \times \mathrm{H}_{\mathrm{S}} / \mathrm{H}_{\mathrm{E}}$.

\subsubsection{Nanoemulsion characteristics}

The droplet size distribution and electrical charge ( $\zeta$-potential) of the nanoemulsions were measured during 16 days of storage time at room temperature. The particle size distribution was measured by static light scattering (SLS) (Mastersizer 2000, Malvern Instruments, Worcestershire, UK). The refractive index of the oil phase (50:50 w/w fish oil:lemon oil), measured with a refractometer, was 1.479 . Samples were diluted in $100 \mathrm{mM}$ 
acetic-acetate buffer ( $\mathrm{pH}$ 3.0) to avoid multiple scattering effects, and then stirred in the dispersion unit of the instrument at a speed of $1250 \mathrm{rpm}$ to ensure they were homogeneous prior to analysis. The particle size is reported as the volume-weighted mean diameter $\left(d_{43}\right)$ in $\mu \mathrm{m}$ (McClements, 2015).

The $\zeta$-potential of the particles was measured by phase-analysis light scattering (Zetasizer NanoZS, Malvern Instruments, Worcestershire, UK). Samples were diluted 1:10 with 100 $\mathrm{mM}$ acetic-acetate buffer solution ( $\mathrm{pH}$ 3.0) and then placed in a capillary cell equipped with two electrodes to assess the electrophoretic mobility of the particles.

\subsubsection{Confocal fluorescence microscopy}

Confocal fluorescence microscopy images were taken to determine destabilization phenomena in the mixed systems. The mixed systems were dyed with Nile red, a fat-soluble fluorescent dye that was previously dissolved at $0.1 \%(\mathrm{w} / \mathrm{v})$ in ethanol. An air-cooled argon ion laser Model IMA1010 BOS (Melles Griot, Carlsbad, CA) was used to excite Nile red at 488 nm. A Nikon Confocal Microscope (Nikon D-Eclipse C1 80i, Nikon, Melville, NY) with a $60 \times$ oil immersion objective lens was used to capture the confocal images. The resulting fluorescent spectra of Nile red were detected in the $515 \mathrm{~nm}$ channel equipped with a narrow pass filter (HQ 515/30 m) with a pinhole size of $150 \mu \mathrm{m}$. The images generated had a size of $512 \times 512$ pixels, with a pixel size of $414 \mathrm{~nm}$, and a pixel dwell time of $61.44 \mu \mathrm{s}$. All images were taken and processed using the instrument software program (EZ- CS1 version 3.8, Nikon, Melville, NY).

\subsubsection{Oxidation measurements}

The concentration of hydroperoxides was used as an indication of the primary oxidation products formed in the nanoemulsions as a result of oxidation, whereas the concentration of thiobarbituric acid reactive substances (TBARS) was used as an indication of secondary oxidation products. A $100 \mathrm{mM} \mathrm{Fe}{ }^{2+}$ solution (prepared from $\mathrm{Fe}_{2} \mathrm{SO}_{4}$ and containing $100 \mathrm{mM}$ EDTA) was incorporated into the mixed systems to achieve a final concentration of $50 \mu \mathrm{M}$ of $\mathrm{Fe}^{2+}$ in the samples. The ferrous solution in mixed systems was added to induce the oxidation reaction and allowing the determination of the antioxidant capacity of sodium alginate. The mixed systems were incubated at $37^{\circ} \mathrm{C}$ in $25-\mathrm{mL}$ plastic tubes during 16 days in the dark.

Hydroperoxides test: The method used to determine hydroperoxides in the nanoemulsions was adapted from a previously reported method (Shantha \& Decker, 1994). A 
0.3-mL aliquot of sample was added to $1.5 \mathrm{~mL}$ of iso-octane/2-propanol blend (3:1), vortexed 3 times for 10 seconds each and successively centrifuged (CL10 centrifuge, Thermo

204 Scientific) for $1 \mathrm{~min}$ at $1000 \mathrm{~g}$. The upper organic phase was collected to determine the 205 hydroperoxide value. A $0.2-\mathrm{mL}$ aliquot of the organic phase was mixed with $2.8 \mathrm{~mL}$ of a 206 methanol:butanol solution $(2: 1), 15 \mu \mathrm{L}$ of $3.94 \mathrm{M}$ thiocyanate solution and $15 \mu \mathrm{L}$ of $0.072 \mathrm{M}$ $207 \mathrm{Fe}^{2+}$ solution. The mixture was vortexed, kept for $20 \mathrm{~min}$ in the absence of light and the 208 absorbance was measured spectrophotometrically (Ultrospec 3000 pro, GE Health Sciences, 209 USA) at $510 \mathrm{~nm}$. The concentration of hydroperoxides was calculated from a standard curve 210 of cumene peroxide.

211 TBARS test: The method used to determine the secondary oxidation products was based 212 on a previously reported method (McDonald \& Hultin, 1987). An aliquot of $1 \mathrm{~mL}$ of sample 213 was added to $2 \mathrm{~mL}$ of the TBA reagent in a screw cap test tube. The TBA reagent was 214 prepared in advance by mixing $100 \mathrm{~mL}$ of a solution containing $20 \% \mathrm{w} / \mathrm{v}$ trichloroacetic acid 215 (TCA), $0.375 \% \mathrm{w} / \mathrm{v}$ thiobarbituric acid (TBA) and $0.25 \mathrm{M} \mathrm{HCl}$ with $3 \mathrm{~mL}$ of $2 \% \mathrm{w} / \mathrm{v}$ of BHT 216 in ethanol. Test tubes were then heated in a boiling water bath for $15 \mathrm{~min}$, cooled down in a 217 water bath, and centrifuged at $1000 \mathrm{~g}$ for $15 \mathrm{~min}$. The absorbance was measured 218 spectrophotometrically in a quartz cuvette at $532 \mathrm{~nm}$. The concentration of secondary 219 oxidation species was determined by means of a 1,1,3,3-tetraethoxypropane (TEP) standard 220 curve.

\subsubsection{Statistical analysis}

The mixing experiments were carried out in duplicate. The measurements of the creaming index and the particle size of the mixed systems were determined in duplicate whereas the rheological properties, $\zeta$-potential, and oxidation tests were carried out in triplicate. The results were expressed as the mean and the standard deviation. A statistical analysis software program (JMP 8, SAS Institute Inc.) was used to perform the analysis of variance. The Student's t test was run to determine significant differences at a 5\%

228 significance level $(\mathrm{p}<0.05)$. 


\section{Results and discussion}

\subsection{Influence of polysaccharide on rheological properties}

The rheological behavior of the samples was characterized by measuring the shear stress versus shear rate dependence, and then calculating the apparent viscosity. Measurements were made for samples containing different amounts of sodium alginate in both the absence and presence of nanoemulsion droplets. The results for the sodium alginate solutions containing no emulsion droplets are shown in Figure 1. Similar results were obtained for the sodium alginate solutions containing nanoemulsion droplets, and therefore this data is not shown. In both cases, the apparent viscosity significantly increased with increasing polysaccharide concentration, which is expected since biopolymers alter the flow profile of aqueous solutions leading to greater energy dissipation. Samples containing relatively high levels of polysaccharide (>0.05\% w/w) showed distinct shear thinning behavior, i.e., the apparent viscosity decreased with increasing shear rate (Figure 1). This effect can be attributed to disentanglement, alignment, and deformation of the polysaccharide molecules within the shear field at high shear rates.

The apparent viscosity (at $10 \mathrm{~s}^{-1}$ shear rate) of aqueous solutions containing different amounts of sodium alginate was compared in the absence and presence of nanoemulsion droplets (Figure 2). These results indicate that the rheology of the mixed systems was dominated by the presence of the alginate molecules, rather than by the nanoemulsion droplets. For both systems, there was a gradual significant increase in shear viscosity with increasing alginate concentration from around 0 to $0.05 \%(\mathrm{w} / \mathrm{w})$, followed by a much steeper increase as the polysaccharide concentration was increased further. Other authors have also reported a steep increase in the viscosity of nanoemulsions with increasing alginate concentration above a certain concentration (Lević, et al., 2015). This increase is a result of stronger chain-chain interactions in more concentrated biopolymer solutions, and tends to lead to distinct non-Newtonian behavior (Manojlovic, Djonlagic, Obradovic, Nedovic, \& Bugarski, 2006). The fact that the apparent viscosity of the sodium alginate solutions containing oil droplets was not significantly different from those containing no oil droplets (Figure 2) can be attributed to the relatively low level of oil droplets $(2.5 \% \mathrm{w} / \mathrm{w})$ present in the mixtures. Other authors have reported an appreciable increase in the apparent viscosity of alginate solutions after mixing with $\mathrm{O} / \mathrm{W}$ emulsions (Sosa-Herrera, Lozano-Esquivel, Ponce de León-Ramírez, \& Martínez-Padilla, 2012). However, these authors used much higher oil 
droplet concentrations $(30 \% \mathrm{w} / \mathrm{w})$ and droplet diameters $(10 \mu \mathrm{m})$ than the ones used in our study.

263

\subsection{Physical stability}

The physical stability of omega-3 nanoemulsions containing sodium alginate was studied by measuring changes in gravitational separation, particle size, $\zeta$-potential, and microstructure.

\subsubsection{Creaming}

The sodium alginate concentration in the aqueous phase had a significant effect on the creaming stability of the omega-3 nanoemulsions (Figure 3). After 24 hours storage, there was evidence of a thin serum layer at the bottom of the nanoemulsions containing intermediate sodium alginate concentrations ( $0.05 \%$ to $0.5 \% \mathrm{w} / \mathrm{w})$. After 48 and 192 hours, the height of the serum layer increased appreciably at these intermediate alginate concentrations, indicating that further droplet creaming had occurred in these samples. At low $(0 \%)$ and high (1\%) alginate concentrations, the degree of creaming was considerably less than that observed at intermediate alginate concentrations. The origin of this effect can be attributed to the ability of the non-adsorbed alginate molecules to induce depletion flocculation, as well as to increase the viscosity of the continuous phase. The lipid droplets were coated by a non-ionic surfactant (Tween 80 ), and therefore there should not have been a strong attraction between the polysaccharide molecules and the droplet surfaces. In the absence of alginate, there would be a strong steric repulsion between the lipid droplets because of the polymeric nature of the hydrophilic surfactant head groups. In the presence of alginate, there will be an osmotic attraction generated between the droplets due to exclusion of the polymer molecules from the vicinity of the droplet surfaces. The strength of this osmotic attraction increases as the concentration of non-adsorbed alginate molecules in the aqueous phase increases. Eventually, the strength of the attractive forces (van der Waals and osmotic) are strong enough to overcome the repulsive interactions (steric) leading to flocculation. At intermediate alginate concentrations the solution viscosity was still relatively low (Figure 2), and so the flocculated droplets tended to cream rapidly because of the increase in particle size. At high alginate concentrations, the droplets were still strongly attracted to each other, but the aqueous phase viscosity was so high that they could not move rapidly upward. Other authors have also reported that non-adsorbed polysaccharides can promote 
droplet aggregation in emulsions through a depletion mechanism at intermediate droplet concentrations (Cao, Dickinson, \& Wedlock, 1990; Cho \& McClements, 2009; Dickinson,

294 Goller, \& Wedlock, 1995; Dickinson, Semenova, Antipova, \& Pelan, 1998; Rebiha, Moulai295 Mostefa, \& HadjSadok, 2012). Besides their concentration, the structure of polysaccharide 296 molecules also impacts their ability to induce depletion flocculation. Theoretical calculations 297 indicate that only small amounts of highly extended polysaccharide molecules are required to 298 induce depletion flocculation in aqueous solutions (McClements, 2000). This would account 299 for the fact that less than $0.1 \%$ sodium alginate was required to promote depletion 300 flocculation in this study.

\subsubsection{Droplet size}

The concentration of sodium alginate added to the nanoemulsions significantly influenced the mean droplet diameter $\left(d_{43}\right)$ and particle size distribution measured after 16 days storage at room temperature (Figures 4 and 5). The initial droplet diameter of the nanoemulsions prepared by microfluidization was $135 \mathrm{~nm}$, and this size remained relatively unchanged throughout storage in the absence of alginate. The addition of sodium alginate to the aqueous phase of the nanoemulsions led to a significant increase in the oil droplet diameter after storage, by an amount that depended on polysaccharide concentration. It should be noted that any flocs present in an emulsion that are held together by osmotic attraction are usually dissociated when the sample is diluted for particle size measurements (McClements, 2015). This phenomenon occurs because dilution causes the polymer concentration to fall below the critical amount required to induce depletion flocculation.

314 Consequently, any observed increase in droplet diameter can be attributed to coalescence, 315 rather than flocculation. The most drastic increase in the mean droplet size was observed at 316 intermediate alginate concentrations, i.e., $0.5 \% \mathrm{w} / \mathrm{w}$ (Figures 4 and 5). These results are in agreement with the creaming stability measurements (Figure 3), indicating that depletion

318 flocculation promoted droplet coalescence. Presumably, the osmotic attraction between the oil droplets was strong enough to force the droplets together and disrupt the interfacial layers that normally separate them. Interestingly, $0.75 \%$ or $1 \%(\mathrm{w} / \mathrm{w})$ sodium alginate led to a more modest increase in droplet size than at intermediate levels. This suggests that high concentrations of polysaccharide may protect the droplets from coalescence, perhaps by retarding their movement, thereby inhibiting their ability to come into close contact. On the 
other hand, nanoemulsions containing $0.05 \%$ (w/w) or lower sodium alginate did not show a remarkable increase in droplet size after storage. In this case, the osmotic attraction between the droplets may not have been strong enough to disrupt the interfacial layers separating them. A number of previous studies have also reported that droplet flocculation can promote droplet coalescence in emulsions by bringing the droplets into close proximity (Dickinson $\&$ Pawlowsky, 1997; Klongdee, Thongngam, \& Klinkesorn, 2012; Ye, Hemar, \& Singh, 2004).

\subsection{3 $\zeta$-potential}

The electrical characteristics of the nanoemulsion droplets were measured during storage to determine if there was any changes in surface charge due to various physicochemical events: generation of ionic lipid oxidation or hydrolysis products (such as free fatty acids); adsorption/desorption of multivalent ions ( $\operatorname{such}$ as $\mathrm{Fe}^{2+}$ ); adsorption of polysaccharide molecules (such as alginate). Overall, the electrical charge ( $\zeta$-potential) on the nanoemulsion droplets was slightly anionic and remained relatively low and constant throughout the storage period (Figure 6). The low charge on the droplets can be attributed to the fact that they are stabilized by a non-ionic surfactant, and so one would not expect a large droplet charge or that the anionic polysaccharide molecules would adsorb to the droplet surfaces. There was a slight increase in the magnitude of the negative charge with increasing alginate concentration. This may have occurred due to cationic ions ( droplet surfaces because they became bound by anionic groups on the non-adsorbed alginate molecules. Alternatively, it may simply have been due to the fact that there were more anionic alginate molecules in the measurement cell of the micro-electrophoresis instrument (Pawar \& Edgar, 2012), which contributed to the signal used to calculate the $\zeta$-potential. However, the micro-electrophoresis technique does not allow one to distinguish the contribution of each species to the overall electrical charge of the system.

\subsubsection{Confocal microscopy}

The microstructure of nanoemulsions containing different amounts of sodium alginate was evaluated after 16 days storage using confocal fluorescence microscopy (Figure 7). The microscopy images of the nanoemulsions confirmed the results of the creaming stability and particle size measurements. In the absence of alginate, the oil droplets were evenly distributed throughout the samples indicating that they were stable to flocculation. At intermediate 
356

357

358

359

360

361

362

363

364

365

366

367

368

369

370

371

372

373

374

375

376

377

378

379

380

381

382

383

384

385

386

387

alginate concentrations $(0.5 \% \mathrm{w} / \mathrm{w})$, the oil droplets were present in large irregularly shaped flocs. The large dimensions of these flocs would account for the high susceptibility of these nanoemulsions to creaming instability (Figure 3). The fact that the droplets were forced into close proximity within the flocs would account for the extensive droplet coalescence observed by particle size analysis (Figure 4). At high alginate concentrations, the oil droplets were still flocculated but the flocs appeared smaller, more tenuous, and more evenly dispersed (Figure

7). The more open structure of the flocs may have been because of the increase in the osmotic attraction between the oil droplets in the presence of high alginate levels. When two or more droplets stick together they tend to remain in their original positions, rather than rolling around, which leads to a more tenuous internal floc structure (lower fractal dimension) (Dickinson, 2013). The smaller size and more even distribution of the flocs may have been due to the relatively high aqueous phase viscosity, which inhibited the movement of individual oil droplets and flocs. Other authors have also used confocal microscopy to monitor changes in the microstructure of emulsions after mixing with biopolymers due to bridging or depletion mechanisms (Moschakis, Murray, \& Biliaderis, 2010; Moschakis, Murray, \& Dickinson, 2006).

\subsection{Lipid oxidation}

Finally, we examined the influence of sodium alginate addition on the oxidative stability of the omega-3 enriched nanoemulsions. Primary oxidation products were monitored by measuring the concentration of hydroperoxides formed during storage, while secondary oxidation products were monitored by measuring the concentration of TBARS formed. An accelerated lipid oxidation protocol was used in this study so that measurements could be carried out in a reasonable timeframe: samples were incubated at $37^{\circ} \mathrm{C}$ and a ferrous solution was added to promote oxidation (Waraho, et al., 2011).

For the sake of comparison, we compared the influence of sodium alginate on lipid oxidation with two other polysaccharides: chitosan and methylcellulose. These three polysaccharides were selected because of their different charge characteristics: alginate (anionic); chitosan (cationic); and, methylcellulose (non-ionic). The influence of polysaccharide addition and type on hydroperoxide formation in the omega-3 nanoemulsions is shown in Figure 8. These nanoemulsions contained either no polysaccharide (control) or $0.1 \%(w / w)$ of sodium alginate, chitosan, or methylcellulose. The concentration of 
hydroperoxides formed progressively increased throughout the 16 days of storage for all the nanoemulsions. Nevertheless, there was slightly less hydroperoxides formed in the nanoemulsions containing chitosan and alginate, than in the one containing methylcellulose or in the control system. Similarly, there was slightly less TBARS form in the nanoemulsions containing chitosan and alginate than in the other two systems (Figure 9). These results suggest that chitosan and alginate were significantly effective at inhibiting lipid oxidation, with chitosan being the most potent antioxidant.

It is well known that transition metals ( promoting lipid oxidation in $\mathrm{O} / \mathrm{W}$ emulsions due to their ability to decompose hydroperoxides located at lipid droplet surfaces into free radicals (Nuchi, McClements, \& Decker, 2001). One would not expect methylcellulose to interfere with this process because it is a non-ionic polysaccharide, and would therefore be incapable of binding transition metals. On the other hand, one would expect that an anionic polysaccharide such as alginate would inhibit lipid oxidation due to its ability to bind cationic transition metals through electrostatic attraction (McClements, et al., 2000). Previous studies have also reported that anionic sodium alginate can inhibit lipid oxidation in fish oil emulsions through this mechanism (Chen, McClements, $\&$ Decker, 2010). The ability of the anionic groups on certain polysaccharides to sequester cationic metal ions has previously been reported in the literature (Nagy \& Szorcsik, 2002). The ability of chitosan to act as an antioxidant must be due to another mechanism, since one would not expect cationic transition metals to bind to the cationic amino groups on the chitosan molecule. Previously, it has been suggested that chitosan may inhibit lipid oxidation in emulsions by adsorbing to the lipid droplet surfaces and forming a positively charged coating that repels cationic metal ions thereby preventing lipid-metal interactions (Klinkesorn, Sophanodora, Chinachoti, McClements, \& Decker, 2005; Panya, et al., 2010). Indeed, studies have shown that cationic chitosan molecules can bind to the surfaces of lipid droplets coated by non-ionic surfactants, due to the presence of a slight negative charge on the droplets (Mun, Decker, \& McClements, 2006). This observation supports the hypothesis that interfacial layer composition and properties play a critical role in determining the rate of lipid oxidation in O/W emulsions (Berton-Carabin, Ropers, \& Genot, 2014). More generally, our results indicate that the charge of a polysaccharide molecule plays an important role in the oxidation of omega-3 fatty acids in emulsions, with both cationic and anionic polysaccharides exhibiting antioxidant activity, but by different physicochemical mechanisms. 


\section{Conclusions}

The results obtained in the present work indicate that addition of sodium alginate to omega-3 enriched nanoemulsions may have either a positive or negative effect on the stability of the system. The addition of sodium alginate caused an appreciable increase in the viscosity of the nanoemulsions, which may be advantageous for some applications (such as dressings, sauces, or dips), but undesirable in other applications (such as nutritional beverages or soft drinks). The stability of the nanoemulsions to gravitational separation depended on the amount of alginate added due to a depletion mechanism. In the absence of alginate, the nanoemulsions were stable to droplet flocculation, coalescence, and creaming due to the large steric repulsion between them. At intermediate alginate levels, the nanoemulsions underwent appreciable flocculation, coalescence, and creaming because of the osmotic attraction generated between the droplets in the presence of non-adsorbed polysaccharide molecules. At high alginate levels, the nanoemulsions were still susceptible to flocculation, but creaming and coalescence were reduced, which was attributed to the ability of the polysaccharide to thicken the aqueous phase and retard droplet and floc movement. The addition of anionic polysaccharide to the nanoemulsions also influenced their stability to lipid oxidation, which was attributed to the ability of the negatively charged carboxyl groups on the alginate molecules being able to bind positively charged transition metals. On the other hand, the addition of a cationic polysaccharide (chitosan) also inhibited the lipid oxidation in the nanoemulsions yet in a lower extent compared to anionic polysaccharide (alginate), however in this case the physicochemical mechanism was different. It was proposed that a layer of cationic chitosan molecules formed around the lipid droplets, which protected them from oxidation by repelling the cationic transition metals in the aqueous phase. The addition of a neutral polysaccharide (methylcellulose) had little impact on the rate or extent of lipid oxidation in the systems. This research shows that ionic polysaccharides may prove to be useful natural antioxidants that can be used to inhibit lipid oxidation in nanoemulsion-based delivery systems. Nevertheless, the system must be carefully formulated to avoid promoting physical instability due to depletion flocculation. 


\section{Acknowledgements}

This material is based upon work supported by the Cooperative State Research, Extension, Education Service, United State Department of Agriculture, Massachusetts Agricultural Experiment Station (Project No. 831), and by the United States Department of Agriculture, NRI Grants (2011-03539, 2013-03795, and 2014-67021).

\section{References}

Adkins, Y., \& Kelley, D. S. (2010). Mechanisms underlying the cardioprotective effects of omega-3 polyunsaturated fatty acids. Journal of Nutritional Biochemistry, 21(9), 781792.

Arab-Tehrany, E., Jacquot, M., Gaiani, C., Imran, M., Desobry, S., \& Linder, M. (2012). Beneficial effects and oxidative stability of omega-3 long-chain polyunsaturated fatty acids. Trends in Food Science \& Technology, 25(1), 24-33.

Akoh, C. C., \& Min, D. B. (2008). Food lipids : chemistry, nutrition, and biotechnology. Boca Raton: CRC Press/Taylor \& Francis Group.

Belhaj, N., Arab-Tehrany, E., \& Linder, M. (2010). Oxidative kinetics of salmon oil in bulk and in nanoemulsion stabilized by marine lecithin. Process Biochemistry, 45(2), $187-$ 195.

Berton-Carabin, C., Genot, C., Gaillard, C., Guibert, D., \& Ropers, M. H. (2013). Design of interfacial films to control lipid oxidation in oil-in-water emulsions. Food Hydrocolloids, 33(1), 99-105.

Berton-Carabin, C. C., Ropers, M.-H., \& Genot, C. (2014). Lipid Oxidation in Oil-in-Water Emulsions: Involvement of the Interfacial Layer. Comprehensive Reviews in Food Science and Food Safety, 13(5), 945-977.

Cao, Y., Dickinson, E., \& Wedlock, D. J. (1990). Creaming and flocculation in emulsions containing polysaccharide. Food Hydrocolloids, 4(3), 185-195.

Chen, B. C., McClements, D. J., \& Decker, E. A. (2010). Role of Continuous Phase Anionic Polysaccharides on the Oxidative Stability of Menhaden Oil-in-Water Emulsions. Journal of Agricultural and Food Chemistry, 58(6), 3779-3784.

Cho, Y.-H., \& McClements, D. J. (2009). Theoretical Stability Maps for Guiding Preparation of Emulsions Stabilized by Protein-Polysaccharide Interfacial Complexes. Langmuir, 25(12), 6649-6657.

Dey, T. k., Ghosh, S., Ghosh, M., Koley, H., \& Dhar, P. (2012). Comparative study of gastrointestinal absorption of EPA \&amp; DHA rich fish oil from nano and conventional emulsion formulation in rats. Food Research International, 49(1), 72-79.

Dickinson, E. (2003). Hydrocolloids at interfaces and the influence on the properties of dispersed systems. Food Hydrocolloids, 17(1), 25-39.

Dickinson, E. (2013). Structure and rheology of colloidal particle gels: Insight from computer simulation. Advances in Colloid and Interface Science, 199, 114-127.

Dickinson, E., Goller, M. I., \& Wedlock, D. J. (1995). Osmotic-pressure, creaming, and rheology of emulsions containing nonionic polysaccharide. Journal of Colloid and Interface Science, 172(1), 192-202. 
Dickinson, E., \& Pawlowsky, K. (1997). Effect of l-carrageenan on flocculation, creaming, and rheology of a protein-stabilized emulsion. Journal of Agricultural and Food Chemistry, 45(10), 3799-3806.

Dickinson, E., Semenova, M. G., Antipova, A. S., \& Pelan, E. G. (1998). Effect of highmethoxy pectin on properties of casein-stabilized emulsions. Food Hydrocolloids, 12(4), 425-432.

Ganesan, B., Brothersen, C., \& McMahon, D. J. (2014). Fortification of Foods with Omega-3 Polyunsaturated Fatty Acids. Critical Reviews in Food Science and Nutrition, 54(1), 98-114.

Gulotta, A., Saberi, A. H., Nicoli, M. C., \& McClements, D. J. (2014). Nanoemulsion-Based Delivery Systems for Polyunsaturated (omega-3) Oils: Formation Using a Spontaneous Emulsification Method. Journal of Agricultural and Food Chemistry, 62(7), 1720-1725.

Kishk, Y. F. M., \& Al-Sayed, H. M. A. (2007). Free-radical scavenging and antioxidative activities of some polysaccharides in emulsions. Lwt-Food Science and Technology, 40(2), 270-277.

Klinkesorn, U., Sophanodora, P., Chinachoti, P., McClements, D. J., \& Decker, E. A. (2005). Increasing the Oxidative Stability of Liquid and Dried Tuna Oil-in-Water Emulsions with Electrostatic Layer-by-Layer Deposition Technology. Journal of Agricultural and Food Chemistry, 53(11), 4561-4566.

Klongdee, S., Thongngam, M., \& Klinkesorn, U. (2012). Rheology and Microstructure of Lecithin-Stabilized Tuna Oil Emulsions Containing Chitosan of Varying Concentration and Molecular Size. Food Biophysics, 7(2), 155-162.

Lević, S., Pajić Lijaković, I., Đorđević, V., Rac, V., Rakić, V., Šolević Knudsen, T., Pavlović, V., Bugarski, B., \& Nedović, V. (2015). Characterization of sodium alginate/dlimonene emulsions and respective calcium alginate/d-limonene beads produced by electrostatic extrusion. Food Hydrocolloids, 45(0), 111-123.

Lorente-Cebrian, S., Costa, A. G. V., Navas-Carretero, S., Zabala, M., Alfredo Martinez, J., \& Moreno-Aliaga, M. J. (2013). Role of omega-3 fatty acids in obesity, metabolic syndrome, and cardiovascular diseases: a review of the evidence. Journal of Physiology and Biochemistry, 69(3), 633-651.

Manojlovic, V., Djonlagic, J., Obradovic, B., Nedovic, V., \& Bugarski, B. (2006). Investigations of cell immobilization in alginate: rheological and electrostatic extrusion studies. Journal of Chemical Technology \& Biotechnology, 81(4), 505-510.

McClements, D. J. (2000). Comments on viscosity enhancement and depletion flocculation by polysaccharides. Food Hydrocolloids, 14(2), 173-177.

McClements, D. J. (2015). Food emulsions principles, practices, and techniques (Third ed.). Boca Raton, FL.: CRC Press.

McClements, D. J., \& Decker, E. A. (2000). Lipid oxidation in oil-in-water emulsions: Impact of molecular environment on chemical reactions in heterogeneous food systems. Journal of Food Science, 65(8), 1270-1282.

McClements, D. J., \& Rao, J. (2011). Food-Grade Nanoemulsions: Formulation, Fabrication, Properties, Performance, Biological Fate, and Potential Toxicity. Critical Reviews in Food Science and Nutrition, 51(4), 285-330.

McDonald, R. E., \& Hultin, H. O. (1987). Some Characteristics of the Enzymic Lipid Peroxidation System in the Microsomal Fraction of Flounder Skeletal Muscle. Journal of Food Science, 52(1), 15-21. 
Moschakis, T., Murray, B. S., \& Biliaderis, C. G. (2010). Modifications in stability and structure of whey protein-coated o/w emulsions by interacting chitosan and gum arabic mixed dispersions. Food Hydrocolloids, 24(1), 8-17.

Moschakis, T., Murray, B. S., \& Dickinson, E. (2006). Particle tracking using confocal microscopy to probe the microrheology in a phase-separating emulsion containing nonadsorbing polysaccharide. Langmuir, 22(10), 4710-4719.

Mun, S., Decker, E. A., \& McClements, D. J. (2006). Effect of molecular weight and degree of deacetylation of chitosan on the formation of oil-in-water emulsions stabilized by surfactant-chitosan membranes. Journal of Colloid and Interface Science, 296(2), 581-590.

Nagy, L., \& Szorcsik, A. (2002). Equilibrium and structural studies on metal complexes of carbohyrates and their derivatives. Journal of Inorganic Biochemistry, 89(1-2), 1-12.

Nuchi, C. D., McClements, D. J., \& Decker, E. A. (2001). Impact of tween 20 hydroperoxides and iron on the oxidation of methyl linoleate and salmon oil dispersions. Journal of Agricultural and Food Chemistry, 49(10), 4912-4916.

Panya, A., Laguerre, M., Lecomte, J., Villeneuve, P., Weiss, J., McClements, D. J., \& Decker, E. A. (2010). Effects of Chitosan and Rosmarinate Esters on the Physical and Oxidative Stability of Liposomes. Journal of Agricultural and Food Chemistry, 58(9), 5679-5684.

Pawar, S. N., \& Edgar, K. J. (2012). Alginate derivatization: A review of chemistry, properties and applications. Biomaterials, 33(11), 3279-3305.

Phillips, G. O., \& Williams, P. A. (2000). Handbook of hydrocolloids. Boca Raton, FL: CRC Press.

Rebiha, M., Moulai-Mostefa, N., \& HadjSadok, A. (2012). Investigations of the Effects of Xanthan and Sodium Caseinate on the Formation and Stability of an Oil-in-Water Emulsion Stabilized by a Nonionic Surfactant Using a Response Surface Method. Journal of Dispersion Science and Technology, 33(1-3), 429-436.

Shahidi, F., \& Miraliakbari, H. (2004). Omega-3 (n-3) fatty acids in health and disease: Part I - Cardiovascular disease and cancer. Journal of Medicinal Food, 7(4), 387-401.

Shahidi, F., \& Miraliakbari, H. (2005). Omega-3 fatty acids in health and disease: part 2 Health effects of omega-3 fatty acids in autoimmune diseases, mental health, and gene expression. Journal of Medicinal Food, 8(2), 133-148.

Shama, F., \& Sherman, P. (1973). Identification of stimuli controlling the sensory evaluation of viscosity II. Oral Methods. Journal of Texture Studies, 4(1), 111-118.

Shantha, N. C., \& Decker, E. A. (1994). Rapid, sensitive, iron-based spectrophotometric methods for determination of peroxide values of food lipids. Journal of Aoac International, 77(2), 421-424.

Sosa-Herrera, M. G., Lozano-Esquivel, I. E., Ponce de León-Ramírez, Y. R., \& MartínezPadilla, L. P. (2012). Effect of added calcium chloride on the physicochemical and rheological properties of aqueous mixtures of sodium caseinate/sodium alginate and respective oil-in-water emulsions. Food Hydrocolloids, 29(1), 175-184.

Sun, Y. E., Wang, W. D., Chen, H. W., \& Li, C. (2011). Autoxidation of Unsaturated Lipids in Food Emulsion. Critical Reviews in Food Science and Nutrition, 51(5), 453-466.

Walker, R., Decker, E. A., \& McClements, D. J. (2015). Development of food-grade nanoemulsions and emulsions for delivery of omega-3 fatty acids: opportunities and obstacles in the food industry. Food \& Function, 6(1), 41-54.

Waraho, T., McClements, D. J., \& Decker, E. A. (2011). Mechanisms of lipid oxidation in food dispersions. Trends in Food Science \& Technology, 22(1), 3-13. 
Ye, A. Q., Hemar, Y., \& Singh, H. (2004). Influence of polysaccharides on the rate of coalescence in oil-in-water emulsions formed with highly hydrolyzed whey proteins. Journal of Agricultural and Food Chemistry, 52(17), 5491-5498. 
Potential antioxidant mechanism of anionic polysaccharides
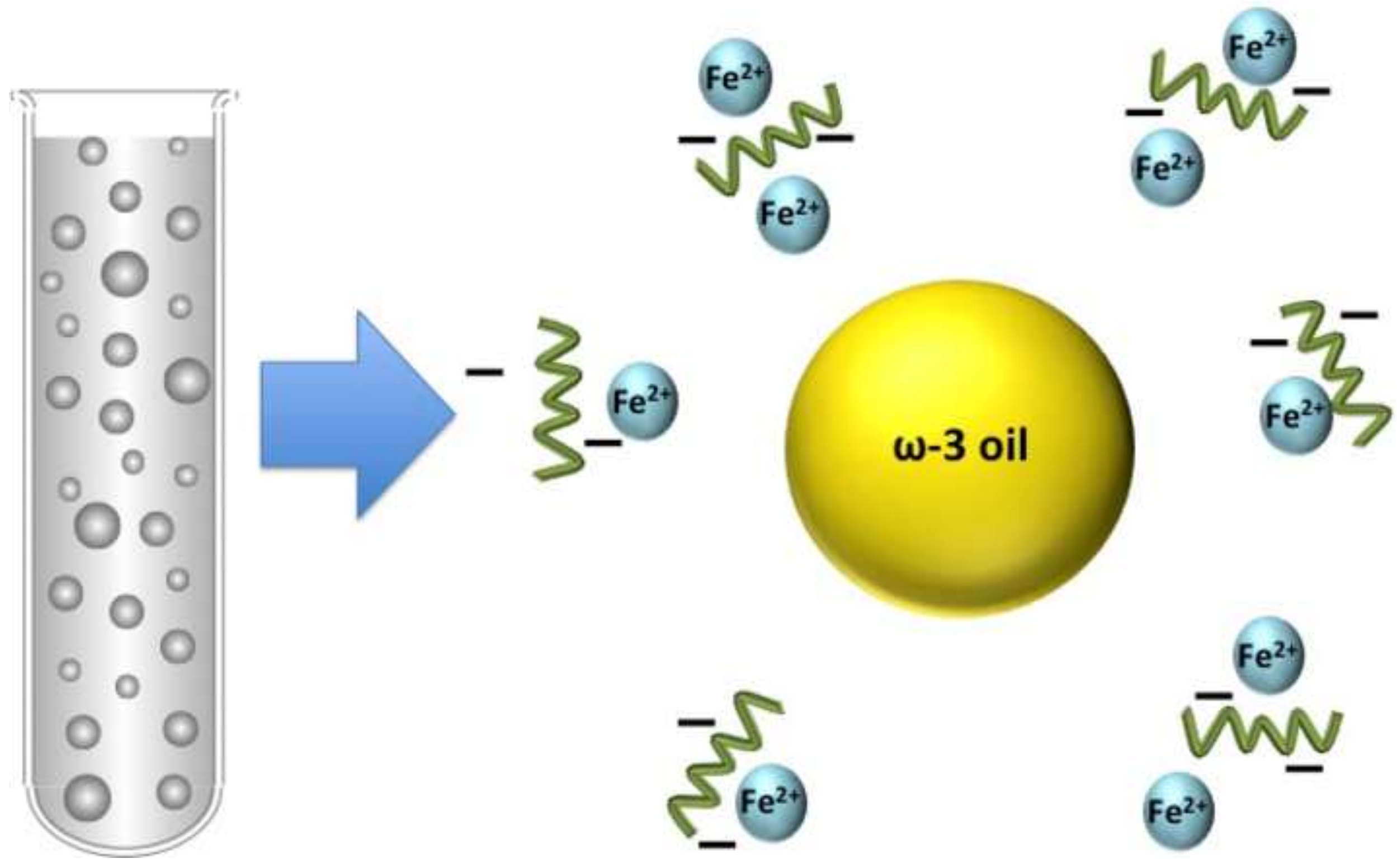


\section{Figure Captions}

Figure 1. Shear stress $(\mathrm{Pa})$ vs. shear rate $\left(\mathrm{s}^{-1}\right)$ of sodium alginate solutions at different concentrations without mixing with omega-3 nanoemulsions

Figure 2. Apparent viscosity (Pa.s) of sodium alginate solutions at different concentrations (\%) with or without mixing with omega-3 nanoemulsions

Figure 3. Creaming index (CI) of omega-3 nanoemulsions after being mixed (24, 48 and 192 hours) with sodium alginate solutions at different concentrations

Figure 4. Droplet size of omega-3 nanoemulsion-sodium alginate mixtures during storage time (16 days)

Figure 5. Particle size distribution of omega-3 nanoemulsion-sodium alginate mixtures immediately after mixing (A) and after 16 days of storage (B)

Figure $6 . \zeta$-potential of omega-3 nanoemulsion-sodium alginate mixtures during storage time (16 days)

Figure 7. Confocal microscopy images of omega-3 nanoemulsions-sodium alginate mixtures after 16 days of storage time

Figure 8. Hydroperoxides concentration (mM) in omega-3 nanoemulsion-sodium alginate mixtures during storage time (16 days)

Figure 9. TBARS concentration $(\mathrm{mM})$ in omega-3 nanoemulsion-sodium alginate mixtures during storage time (16 days) 


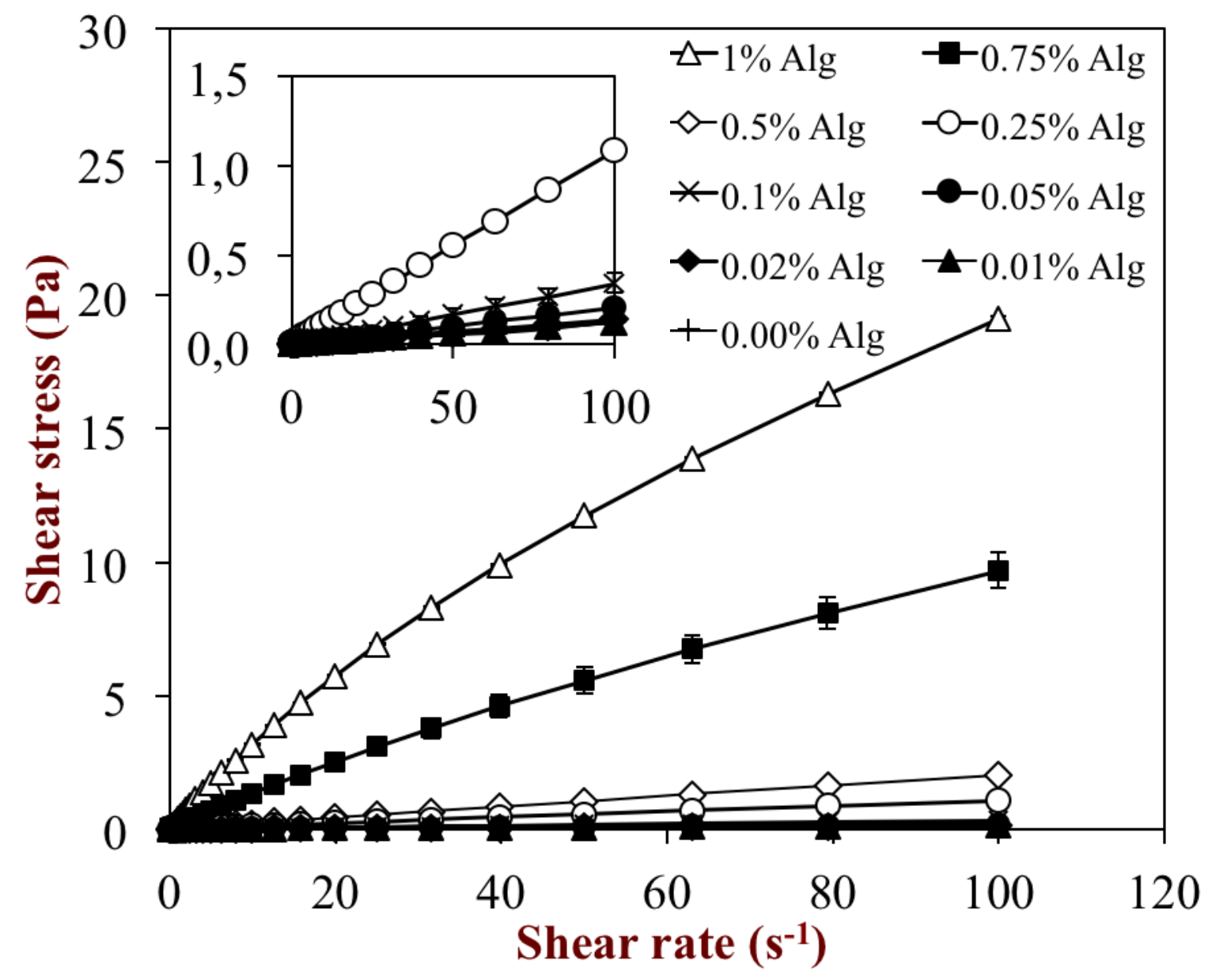




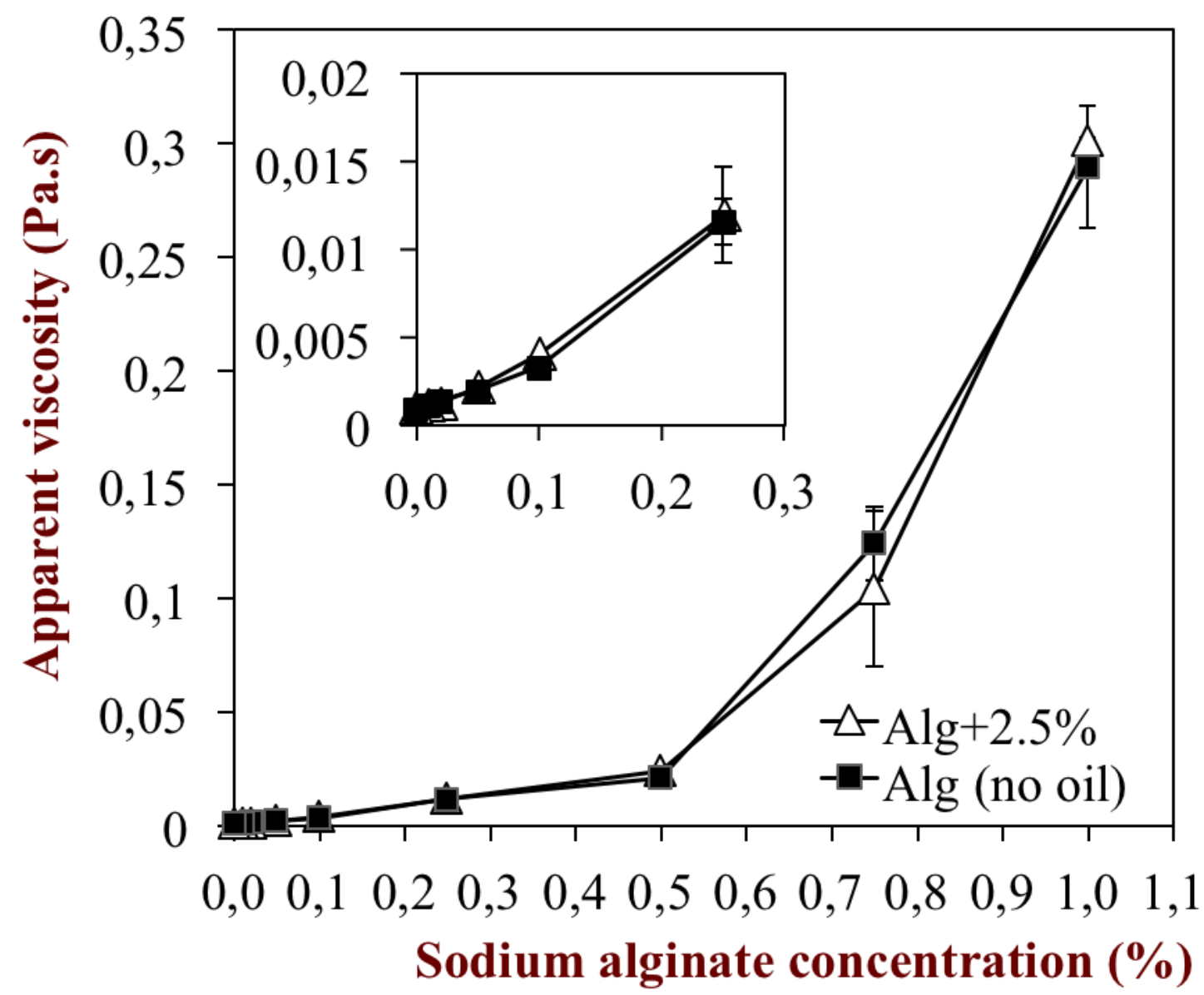


Figure 4

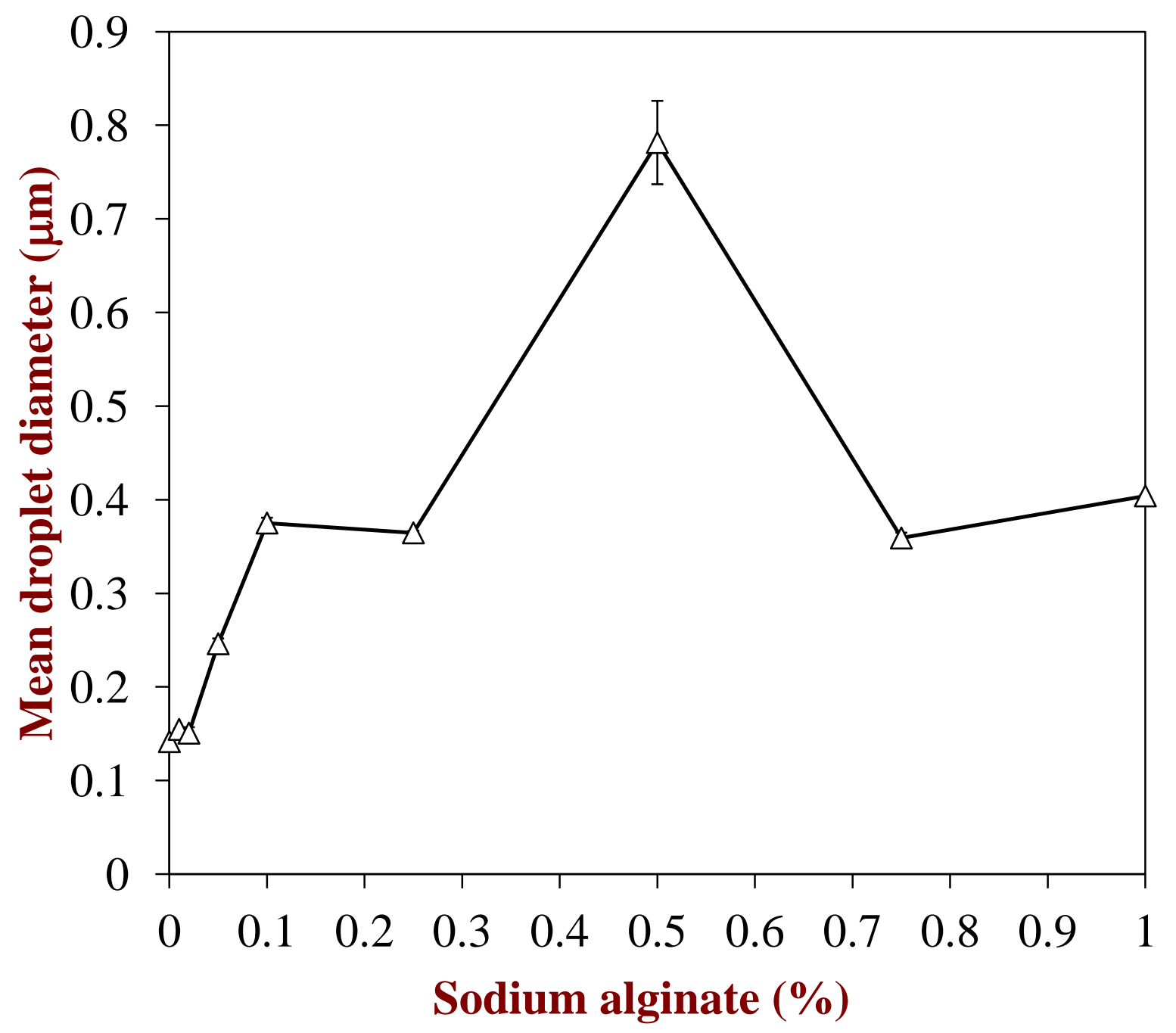


Figure 5

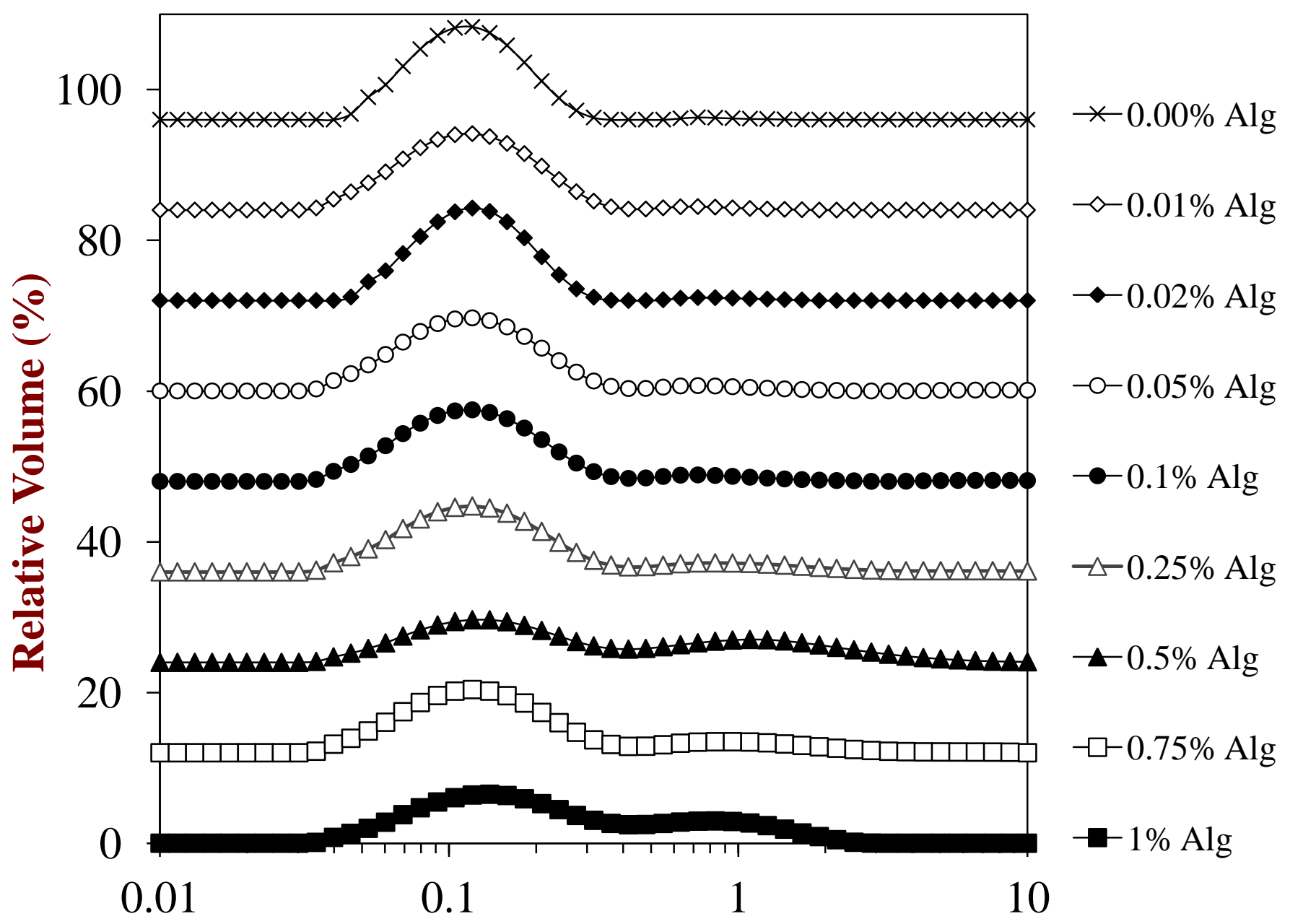

Particle Diameter $(\mu \mathrm{m})$ 


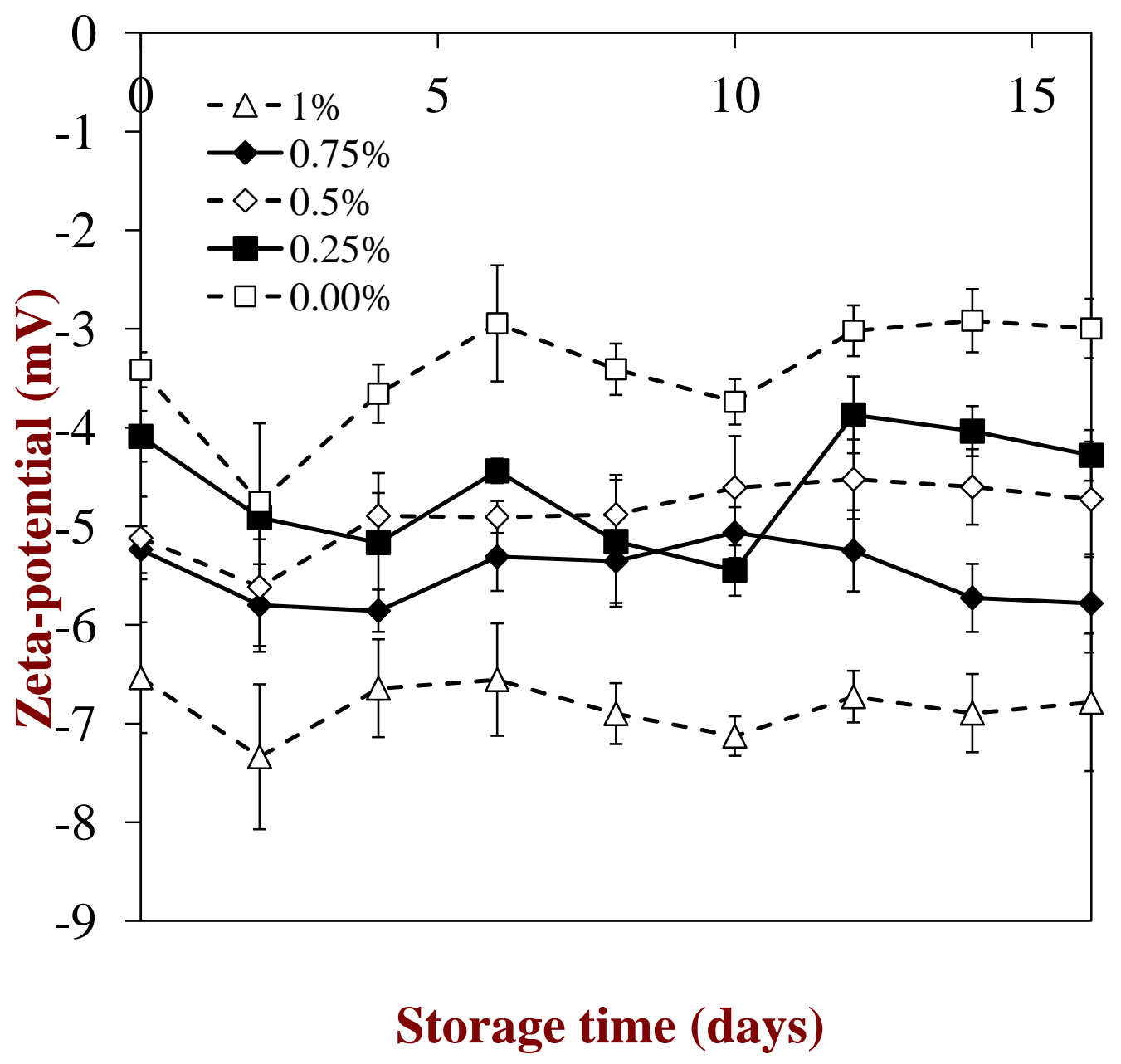




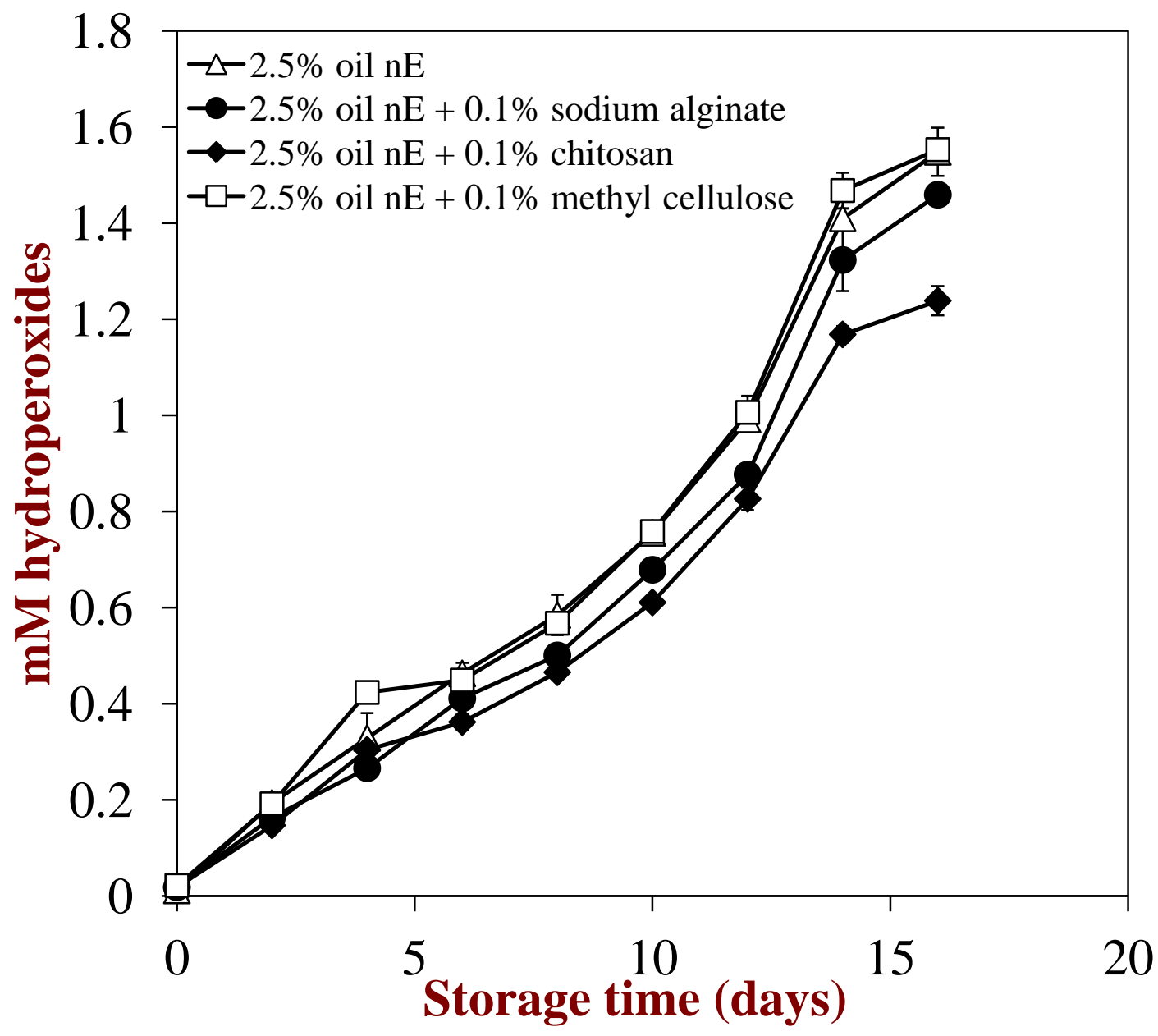




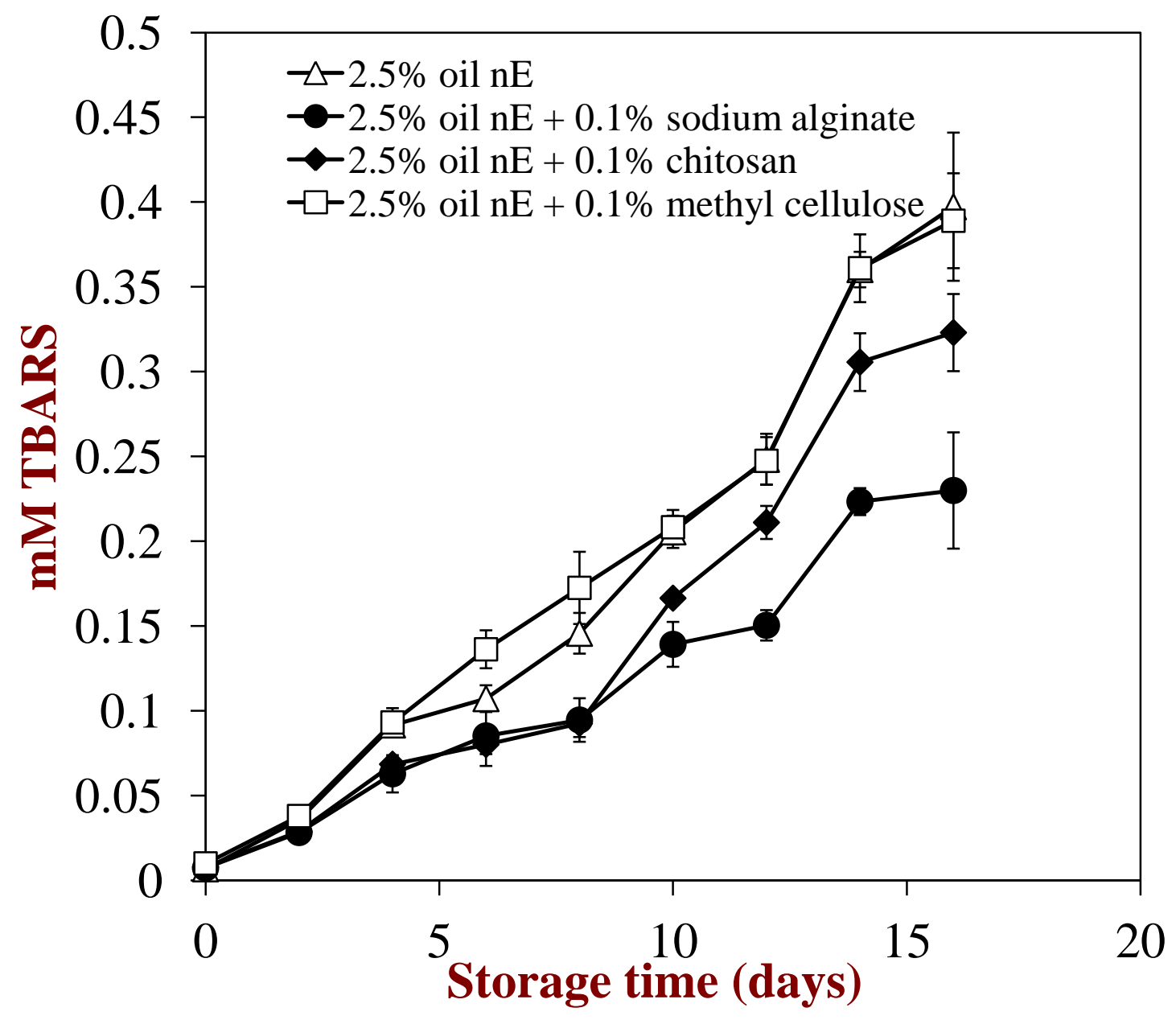

